\title{
Light Absorption Enhancement in Organic Solar Cell by Embedding Ag Nanoparticles and Nanochains within the Active Layer
}

\author{
Sheng-Qing Zhu, ${ }^{1,2,3}$ Tong Zhang, ${ }^{1,2,3}$ Xin-Li Guo, ${ }^{4}$ Feng Shan, ${ }^{1,2,3}$ and Xiao-Yang Zhang ${ }^{1,2,3}$ \\ ${ }^{1}$ School of Electronic Science and Engineering, Southeast University, Nanjing 210096, China \\ ${ }^{2}$ Key Laboratory of Micro-Inertial Instrument and Advanced Navigation Technology, Ministry of Education, Nanjing 210096, China \\ ${ }^{3}$ Suzhou Key Laboratory of Metal Nano-Optoelectronic Technology, Suzhou Research Institute of Southeast University, \\ Suzhou 215123, China \\ ${ }^{4}$ School of Materials Science and Engineering, Southeast University, Nanjing 211189, China
}

Correspondence should be addressed to Tong Zhang; tzhang@seu.edu.cn

Received 28 February 2014; Accepted 26 March 2014; Published 23 April 2014

Academic Editor: Jing Chen

Copyright (c) 2014 Sheng-Qing Zhu et al. This is an open access article distributed under the Creative Commons Attribution License, which permits unrestricted use, distribution, and reproduction in any medium, provided the original work is properly cited.

\begin{abstract}
We numerically investigate the light absorption enhancement of organic photovoltaic cells by embedding Ag nanoparticles and nanochains within the active layer using a finite element method. We analyze the enhancement mechanism of light absorption and systematically study the influence of factors such as the size and the period of silver nanoparticles. The result shows the localized surface plasmon resonance of the particles has a significant influence on the light absorption. Under AM1.5 illumination condition, a relative enhancement with a factor of $107.1 \%$ is observed for nanoparticles with a diameter of $30 \mathrm{~nm}$ and a period of $200 \mathrm{~nm}$. In addition, different types of nanochain structures have been studied, and we find that, comparing to monodisperse nanoparticles, nanochain structures can further enhance the light absorption because of the stronger light harvesting in the long wavelength range of $600-800 \mathrm{~nm}$.
\end{abstract}

\section{Introduction}

Organic solar cells (OSCs) based on conjugated polymer and fullerene composites, because of the various advantages, such as lightweight, solution processed, mechanical flexibility, and large-area coverage, have been intensively studied as a potential new candidate for solar energy conversion system [1-4]. However, comparing to conventional solar cells, including silicon, GaAs, and $\mathrm{CuIn}_{x} \mathrm{Ga}_{1-x} \mathrm{Se}_{2}$ [5-7], the OSCs have got relatively lower energy conversion efficiency and can not compete with conventional energy. This demerit is mainly ascribed to the poor light absorption of the active layer in the OSCs. In order to increase the light absorption of the OSCs, the thickness of active layer, usually being a few hundred nanometers $(30-150 \mathrm{~nm})$ [8-10], should be increased in conventional method. However, the low chargecarrier mobility and small exciton diffusion length of most molecular and polymeric materials limit the increase of the active layer thickness in OSCs [11, 12]. If the active layer thickness increases, this will in turn result in insufficient carrier generation and low power conversion efficiency.

To overcome the poor light absorption, several light trapping strategies may be explored in the design of OSCs to improve the power conversion efficiency. For instance, Kim et al. utilized solution-based titanium oxide as an optical spacer to harvest more sunlight [13]; Sista et al. employed tandem structure to improve the light absorption [8]. Recently, researchers proposed a more novel approach that uses noble metallic nanostructures to obtain effective light trapping for thin-film solar cells $[14,15]$. These nanostructures can excite localized surface plasmon resonances (LSPRs), arising from the collective oscillation of conduction electrons in noble metal nanoparticles when the electrons are disturbed from their equilibrium positions, and usually served as a local 


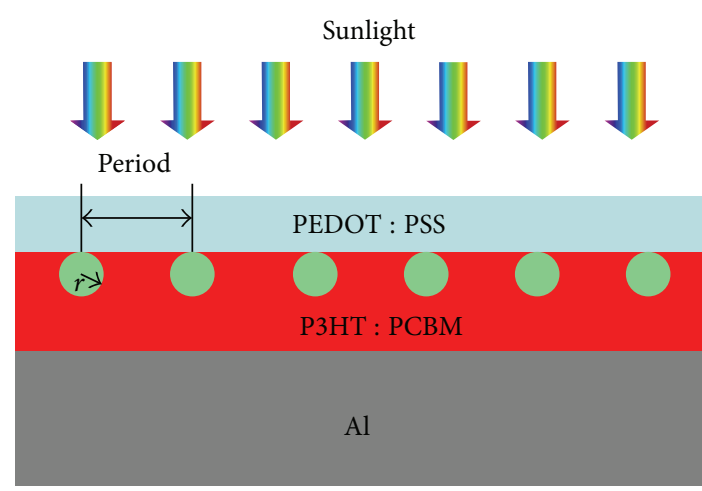

(a)

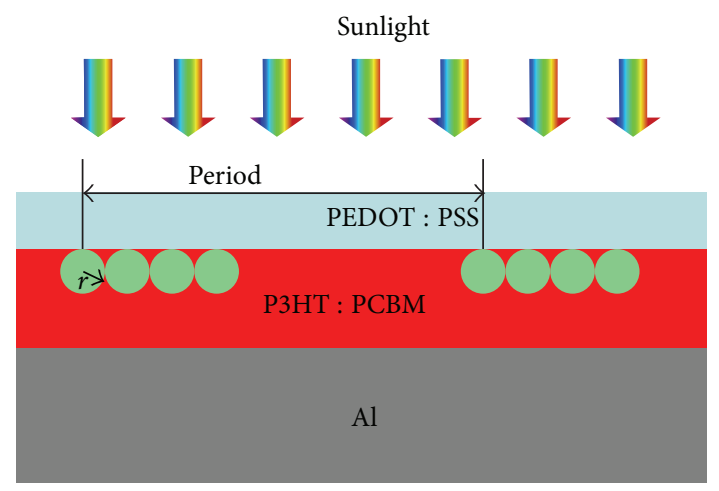

(b)

FIgURE 1: (Color online) Schemes of the simulation OSCs' models embedded with monodisperse periodic SNPs (a) and SNCs composed of adjacent SNPs (two, three, four, or five; only the case of four was shown).

field enhancer or a light scattering center depending on the size of metal nanoparticles [16]. Up to now, numerous experiment studies on the influence of noble metallic nanostructures in inorganic solar cells have been performed, which involved nanoislands $[17,18]$, spheres, nanorods, nanostars [14], nanogrids $[19,20]$, and so forth. However, comparing to experiment investigation, theoretical study especially of the enhancement mechanism of light absorption has not been well developed. Duche et al. studied the light absorption enhancement induced by silver nanoparticles (SNPs) within the OSCs by finite-difference time-domain (FDTD) method [21]; however, they only modeled a thin film of active layer with nanoparticles in three-dimensional simulations. Shen et al. modeled a multilayer structure with silver nanoparticles imbedded in the middle of the active layer [22], but this structure is difficult to realize in experiment because the preparation process that adds water solvable nanoparticles into photovoltaic material solution (chlorobenzene or chloroform as the solvent) usually is incompatible or complicated.

In this work, we model a more realistic solar cell structure with cathode, active layer, and anode based on the finite element method (FEM). In this model, periodic monodisperse SNPs and silver nanochains (SNCs) composed of adjacent nanoparticles, used as light absorption enhancement structures, are placed in the interface between conductive polymer layer and active layer. Silver is chosen since it allows for low metal absorption loss and it also has a beneficial surface plasmon resonance wavelength suitable for enhancing the P3HT: PCBM OSC. We systematically investigate the effect of numerous factors, such as the size, period of monodisperse SNPs, and the length of SNCs, on the light absorption of the OSCs. Furthermore, the enhancement mechanism of light absorption is analyzed. The result shows that the origin of the light absorption enhancement should be attributed to near electric field enhancement of the nanostructures.

\section{Simulation Models and Methods}

We considered the OSCs layout with the schematic cross section shown in Figure 1. Due to the indium scarcity, an indium tin oxide- (ITO-) free structure [23] was used and the ITO was replaced by highly conductive polymer, poly (3, 4-ethylenedioxythiophene) : poly (styrenesulfonate) (PEDOT: PSS) with $30 \mathrm{~nm}$ thickness, which is a polymer with good thermal and chemical stability and good flexibility. For the active layer, the typical photovoltaic material, poly (3exylthiophene): (6,6)-phenyl-C61-butyric-acid-methyl ester (P3HT: PCBM), with 1:1 weight ratio was used. Aluminum film with the thickness of $120 \mathrm{~nm}$ was used as the cathode. In this work, the light absorption structures include two types: periodic monodisperse SNPs (Figure 1(a)) and periodic SNCs (Figure 1(b)). The refractive index of PEDOT: PSS, silver, aluminum, and P3HT: PCBM are taken from the literature $[18,24]$. Meanwhile, in order to save computation time and memory, we reduce the three-dimensional (3D) simulation to a two-dimensional (2D) one because $2 \mathrm{D}$ model can give similar results to a 3D model according to the early literature reported [25].

In this simulation, the calculation of light absorption is carried out by $2 \mathrm{D}$ finite element method (FEM). The FEM, which is a popular numerical approach for the solution of many engineering problems, is currently recognized as a powerful tool for the analysis of several optical structures, particularly those with arbitrary shapes, index profiles, nonlinearities, and anisotropies. We assume the light illumination on the device as incident plane waves under TM polarization with wavelengths of $300-800 \mathrm{~nm}$, which is the region of interest for the P3HT: PCBM material. Periodic boundary conditions are set at the left and right boundaries, while perfectly matched layer (PML) absorbing boundary conditions are used at the top and bottom boundaries of the computational domain. In this paper, the absorption $A(\lambda)$ in different parts of OSCs (in active layer, SNPs or SNCs) is calculated by integrating the divergence of the Poynting vector $\vec{S}$ (power flow) which is then normalized with input power $P_{\text {inc }}$ :

$$
A(\lambda)=\frac{\int \nabla \cdot \vec{S} d V}{P_{\text {inc }}},
$$

in which $V$ refers to the total volume of integration. 


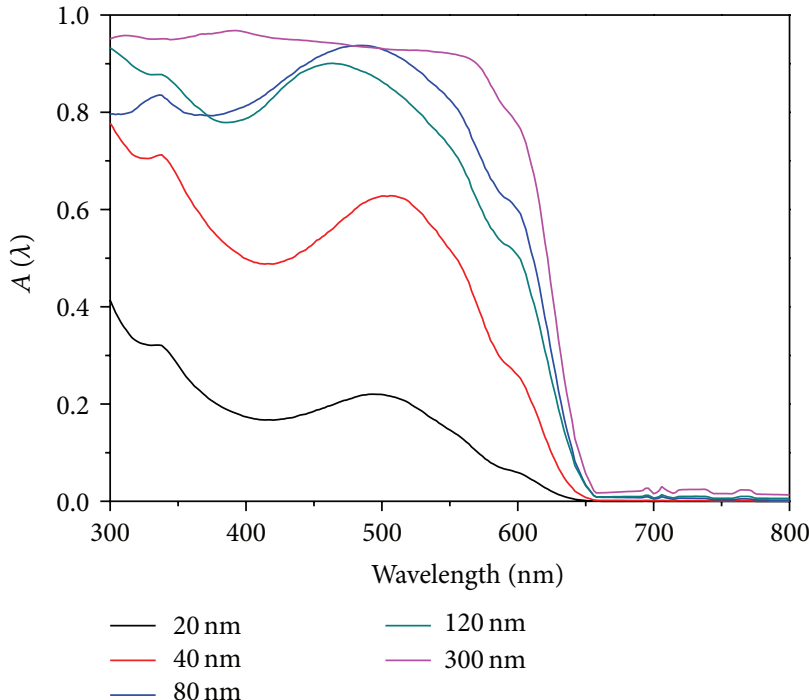

(a)

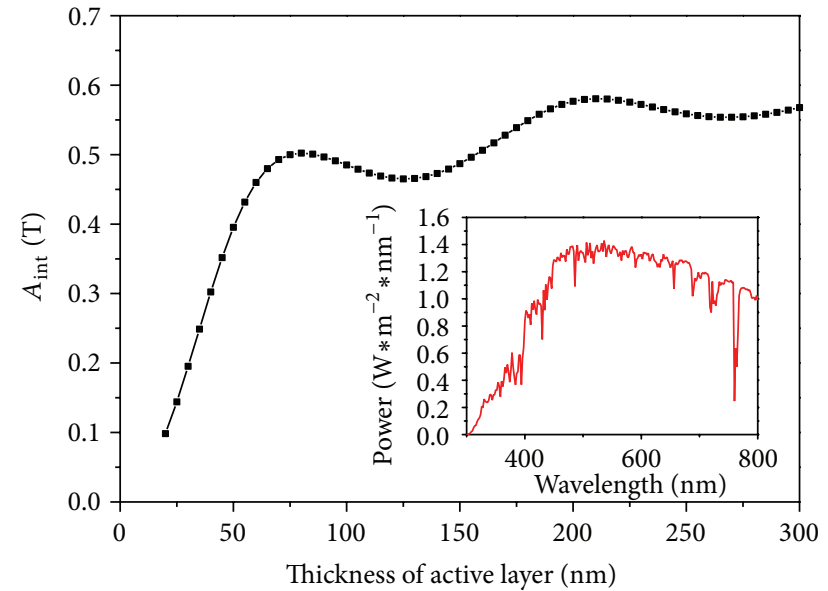

(b)

FIgURE 2: (Color online) Calculations for pristine OSCs without plasmonic structures. (a) Absorption spectra $A(\lambda)$ of active layer with different thicknesses $T$. (b) Integrated absorption $A_{\text {int }}$ as a function of film thickness ( $T$ ) for normal incidence (inset is AM1.5 solar spectrum).

To quantify the absorption capability of our OSC models in the wavelength region of interest wavelength (300$800 \mathrm{~nm}$ ), we define a normalized weighted integrated absorption for the AM1.5G spectrum:

$$
A_{\text {int }}=\frac{\int\left[P_{\mathrm{AM} 1.5 \mathrm{G}}(\lambda) \times A(\lambda)\right] d \lambda}{\int P_{\mathrm{AM} 1.5 \mathrm{G}}(\lambda) d \lambda} .
$$

\section{Results and Discussion}

3.1. Absorption Property of Pristine OSCs. Firstly, we calculate the simulation model for pristine OSCs without any silver nanostructures and investigate the influence of the active layer thickness on the absorption. The absorption spectra of active layer are shown in Figure 2(a). On the whole, these simulation spectra matched the experimental data well [26, 27]. The three apparent vibronic absorption shoulders near the wavelengths of $500 \mathrm{~nm}, 550 \mathrm{~nm}$, and $600 \mathrm{~nm}$ observed from the absorption spectra can be attributed to the strong interchain-interlayer interactions of P3HT [28]. The calculated results proved that the model program is accurate and useful. It also can be obviously seen that the OSCs with thicker active layer harvest more incident sunlight. The values of absorption near $500 \mathrm{~nm}$ with $20 \mathrm{~nm}, 40 \mathrm{~nm}, 120 \mathrm{~nm}$, and $300 \mathrm{~nm}$ active layer thicknesses are $22 \%, 62 \%, 87 \%$, and $93 \%$, respectively. However, it is interesting to note that the absorption spectrum with an $80 \mathrm{~nm}$ thick active layer is more intense than that with a $120 \mathrm{~nm}$ thick active layer in the wavelength range of $370 \mathrm{~nm}$ to $800 \mathrm{~nm}$. To explore the reason, we calculated absorption spectra with other thicknesses $(20-300 \mathrm{~nm})$ of active layer and integrated the absorption spectra weighted by the AM1.5G solar spectrum (inset in Figure 2(b)). Figure 2(b) shows the integrated absorption $A_{\text {int }}$ as a function of the active layer thickness for normal incidence and there are two absorption peaks near $75 \mathrm{~nm}$ and $200 \mathrm{~nm}$. These absorption peaks can be ascribed to interference effects of the two interfaces (also called FabryPerot effects) of the active layer when sunlight irradiates on the OSCs. This result matched well with previous works and this is why the absorption intensity with $80 \mathrm{~nm}$ active layer thickness is stronger than that with $120 \mathrm{~nm}$ [22].

Obviously, increase of the active layer thickness can promote the incident light absorption. However, as the thickness increases, carrier recombination will become significant, which reduces the external quantum efficiency of the OSCs. To resolve this problem, we embed SNPs into the interface between active layer and buffer layer as absorption enhancement structures to harvest more incident light. This kind of OSCs with SNPs could combine the advantages of thin and thick active layers, in which both high carrier collection efficiency and strong light harvesting can be kept.

3.2. OSCs Embedded with Periodic Monodisperse SNPs. In this section, we systematically investigate the optical property of OSCs embedded with periodic SNPs. In the simulation model, the particles size and the thickness of active layer are set as $D$ (diameter) $=10 \mathrm{~nm}$ and $T=60 \mathrm{~nm}$, respectively. The absorption spectra of the active layer which includes P3HT: PCBM and periodic monodisperse SNPs are shown in Figure 3(a), where a broadband absorption enhancement can be observed in the wavelength range of $300-800 \mathrm{~nm}$ for all periods. It is also found that the absorption enhancement value is associated with the period. The values of absorption in OCSs near $500 \mathrm{~nm}$ with the periods of $30 \mathrm{~nm}, 60 \mathrm{~nm}$, $90 \mathrm{~nm}, 150 \mathrm{~nm}$, and $450 \mathrm{~nm}$ are $93.4 \%, 91.5 \%, 90.5 \%, 89.6 \%$, and $88.3 \%$, respectively. However, these absorption values contain the contribution of the periodic SNPs. In order to calculate the net absorption of $\mathrm{P} 3 \mathrm{HT}$ : PCBM, the absorption 


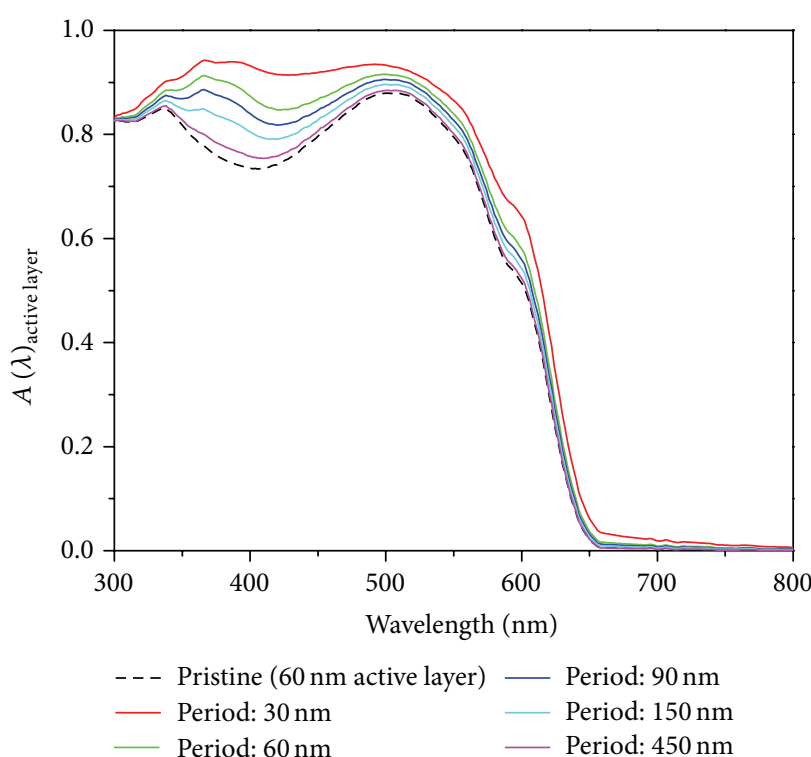

(a)

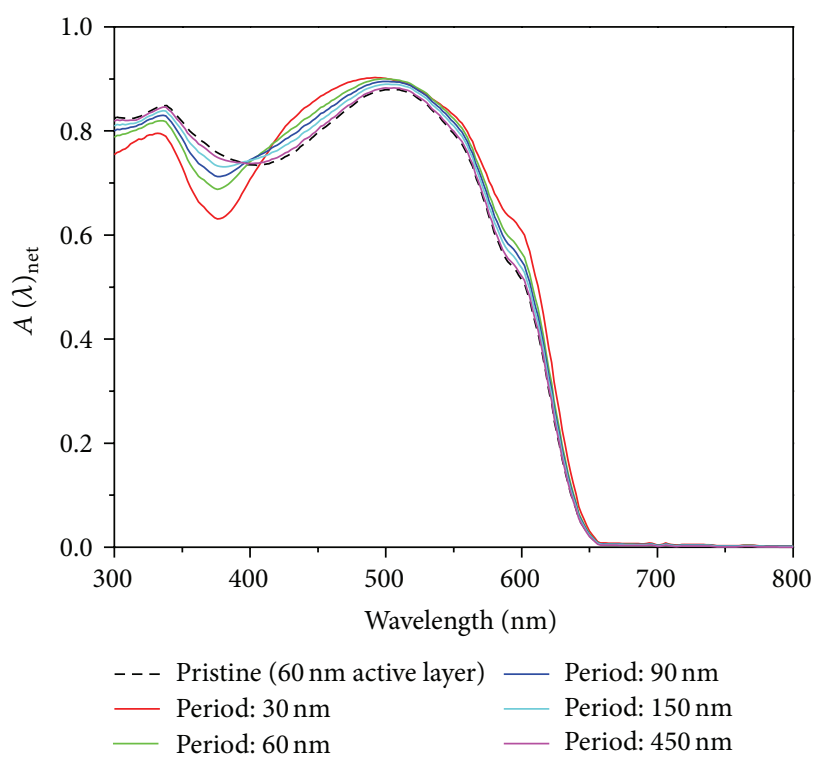

(c)

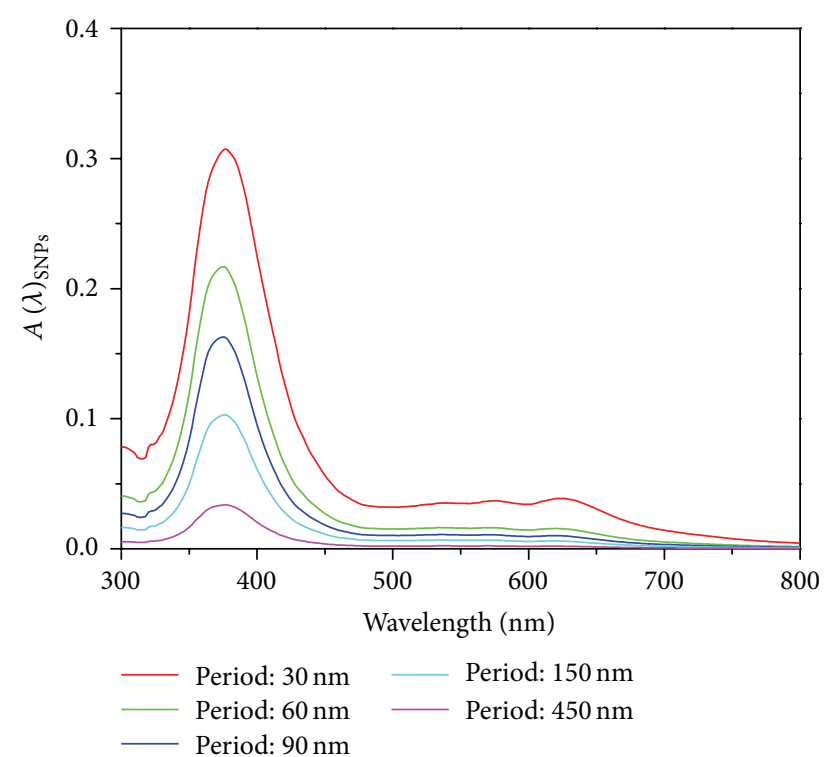

(b)

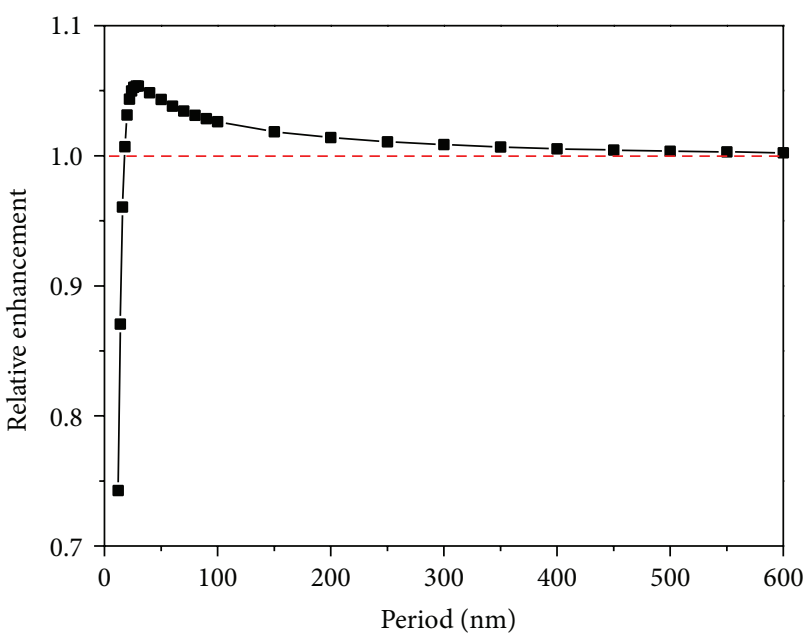

(d)

FIgure 3: (Color online) Simulation results for OSCs embedded with monodisperse SNPs with different periods. (a) Absorption spectra of active layer (include SNPs); (b) absorption spectra of SNPs; (c) net absorption spectra of active layer (exclusive of SNPs); (d) relative enhancement as a function of particle periods (the red dash line refers to the value of corresponding pristine OSC).

spectra of SNPs are calculated and shown in Figure 3(b). The absorption peaks near $380 \mathrm{~nm}$ are attributed to dipole resonance, which was caused by the alternating electric field on the SNP surfaces when incident light irradiated on the OSCs [29]. Figure 3(c) exhibits the net light absorption of P3HT : PCBM (exclusive of SNPs). The main difference of the spectra from Figure 3(a) is that light absorption enhancement appears only in part of the wavelength range of 403$800 \mathrm{~nm}$ and light absorption weakens in 300-402 $\mathrm{nm}$ ranges. To achieve the whole absorption enhancement in OSCs embedded with SNPs, the integrated absorption weighted by AM1.5G irradiation was obtained (not exhibited) and relative enhancement (ratio of $A_{\text {int }}$ with SNPs to that without SNPs) in the active layer is shown in Figure 3(d). The result shows the light absorption enhancement as a function of the period of SNPs and there exists an optimum period of $30 \mathrm{~nm}$ maximizing the absorption enhancement with a factor of around $105.37 \%$. Meanwhile, we also notice that the relative enhancement dropped below 1 when active layer thickness is less than $18 \mathrm{~nm}$. This is because the volume of P3HT: PCBM is reduced and luminous flux decreases when OSCs are embedded with high concentration-density SNPs.

It is well known that the localized plasmon resonance in SNPs can cause near-field enhancement and enhanced 


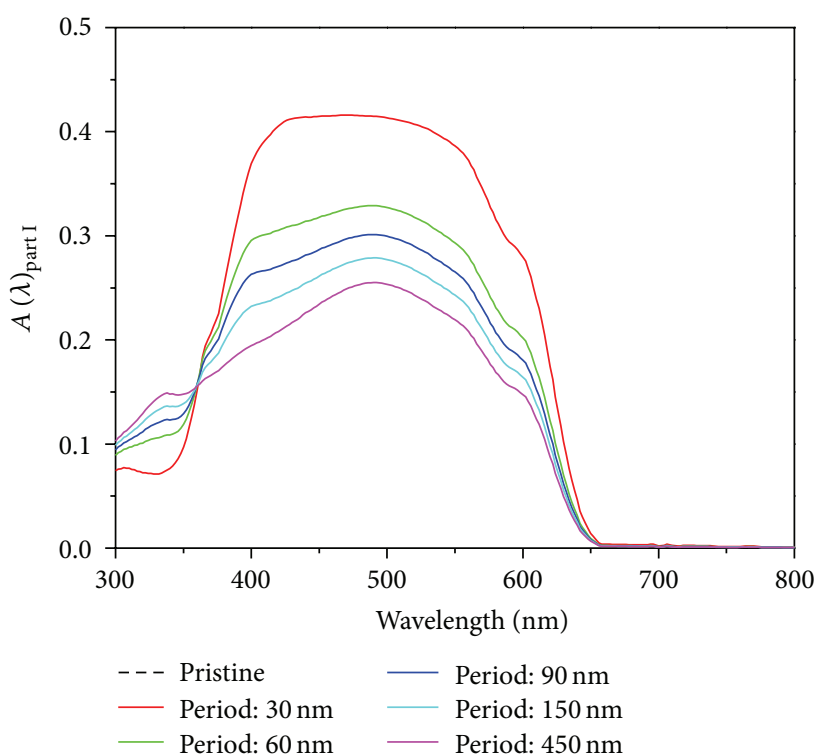

(a)

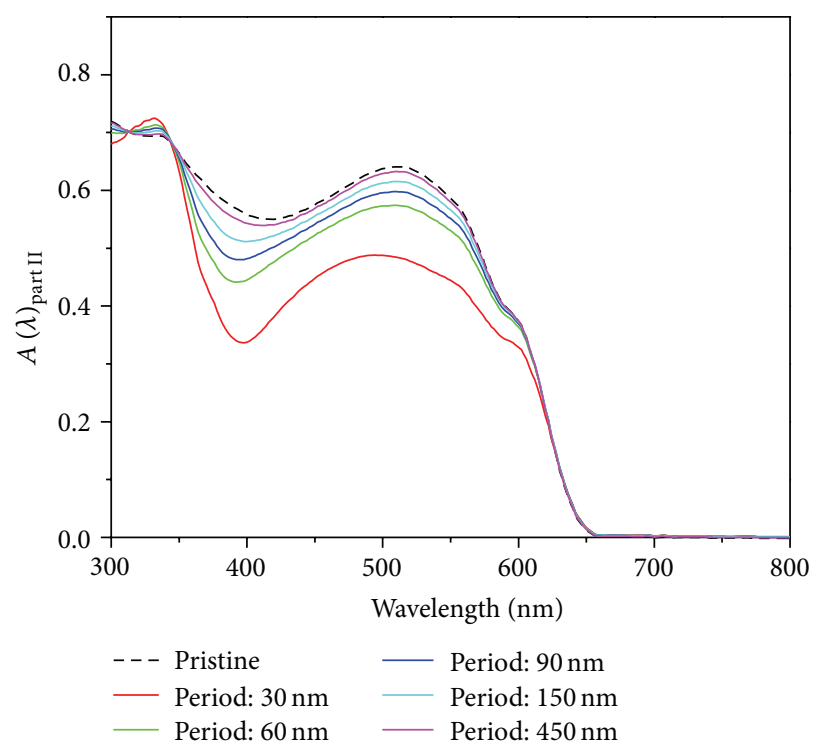

(c)

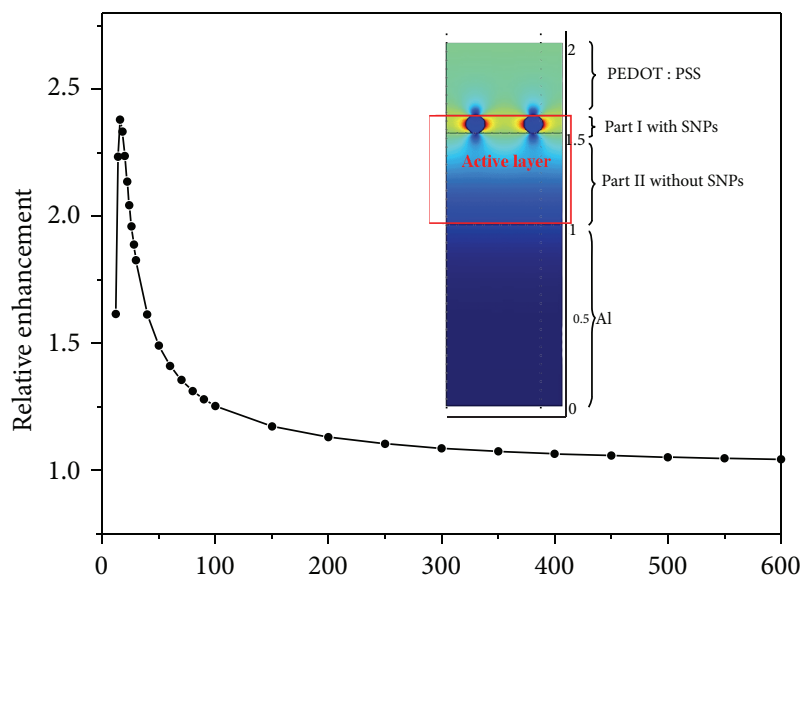

(b)

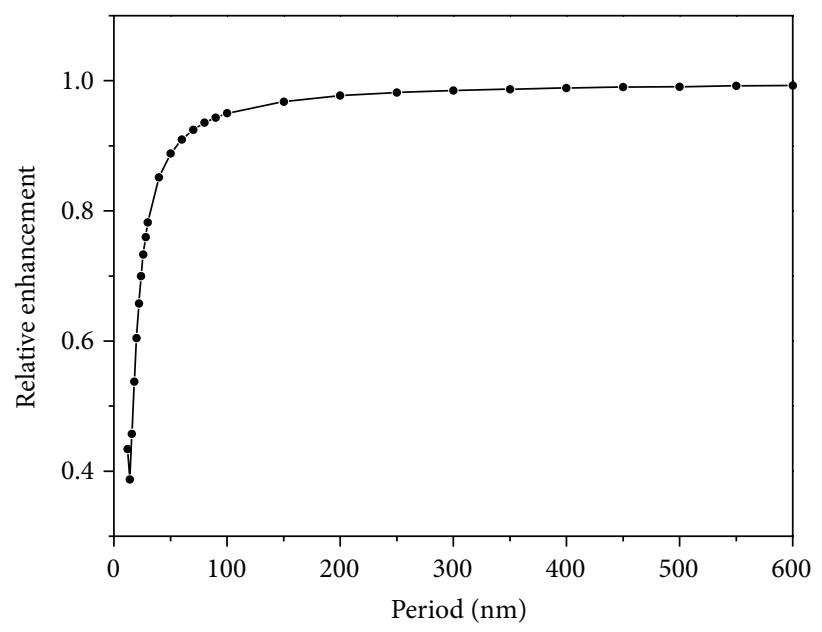

(d)

FIGURE 4: (Color online) (a) Absorption spectra of active layer in the region of part I; (b) relative enhancement for the region of part I; the inset shows the OCS model profile; (c) absorption spectra of active layer in the region of part II; (d) relative enhancement for the region of part II.

scattering cross section, and both of them can be the mechanism to enhance the absorption in a solar cell in previous works. In order to further explore which mechanism of absorption enhancement exists in OCS embedded with SNPs, the active layer was divided into two regions (part I and part II). The region of part I contains the sublayer embedded with SNPs and the region of part II contains the other active layer (see the inset in Figure 4(b)). Figure 4(a) shows the net absorption spectra of $\mathrm{P} 3 \mathrm{HT}$ : $\mathrm{PCBM}$ in the region of part I. The absorption spectra exhibit a broadband enhancement in the range between around 362 and $650 \mathrm{~nm}$ comparing to that in corresponding region of pristine OSC.
Figure 4(b) shows the relative enhancement with different periods and the highest factor can reach to $238.1 \%$ in the region of part I. However, for the other region (part II), there is no light absorption enhancement at any wavelength (Figure $4(\mathrm{c})$ ) and the enhancement factor drops below 1 (Figure 4(d)). Contrasting the optical properties in the two regions, we can conclude that only the region containing SNPs can obtain obvious light absorption enhancement. The phenomenon also implies that the absorption enhancement is mainly attributed to near electric field enhancement and not to enhanced scattering effects; this is because only in the region of part I the electric field in the gap region 


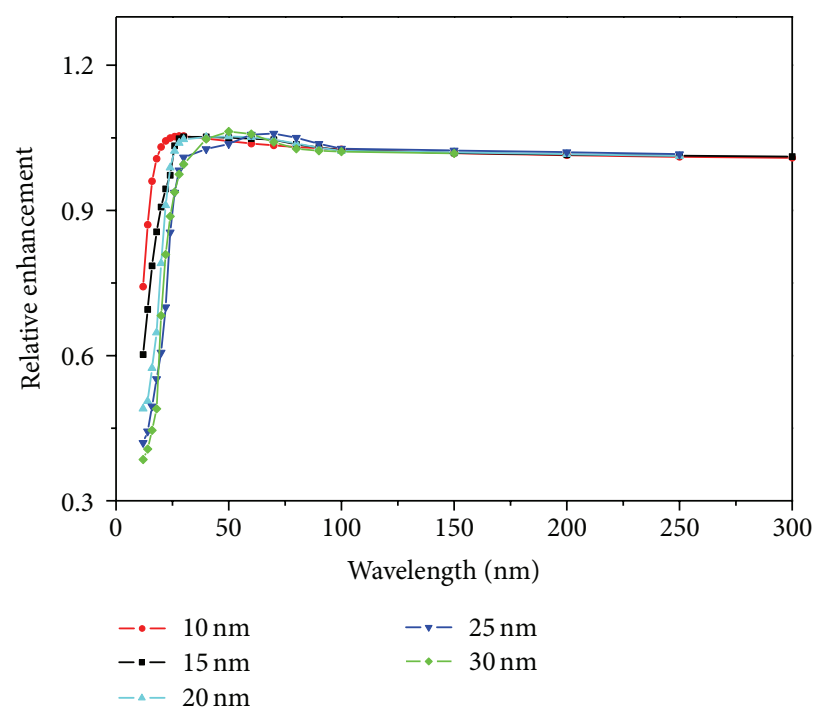

(a)

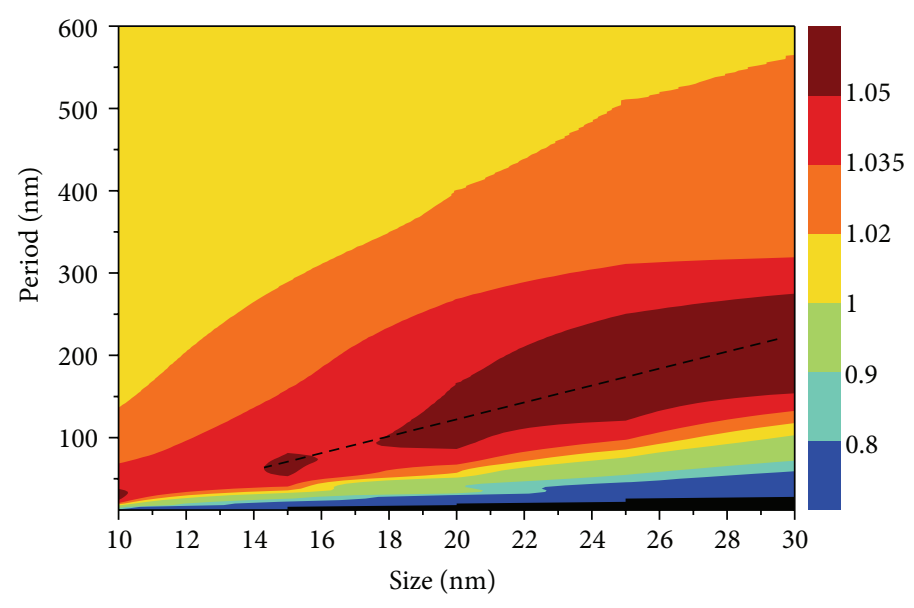

(b)

Figure 5: (Color online) (a) Relative enhancement using different SNPs with the size of 10, 15, 20, 25, and 30 nm; (b) relative enhancement in active layer for various particle sizes $(D)$ and periods $(P)$.

between SNPs can be sharply enhanced by several orders of magnitude. Therefore, these SNPs are used as nanoantennas to increase the light absorption in this simulation model.

Apart from the case of particle size of $10 \mathrm{~nm}$, we also calculate other OSC models which are embedded with SNPs with the size of $15,20,25$, and $30 \mathrm{~nm}$ to achieve the optimal particle size $D$ and period $P$ of the SNP structures. Figure 5(a) shows relative enhancement in the whole active layer for various particle sizes $(D)$ and periods $(P)$. These enhancement factors exhibit similar tendency as the particle size of $10 \mathrm{~nm}$ and highest factors as large as $105.2 \%, 105.3 \%, 105.9 \%$, and $107.1 \%$ are observed for particle size of $15,20,25$, and $30 \mathrm{~nm}$, where the corresponding optimal periods are $72 \mathrm{~nm}$, $115 \mathrm{~nm}, 158 \mathrm{~nm}$, and $200 \mathrm{~nm}$, respectively. From Figure 5(a), it is also found that OSC with big particle size usually needs large period to obtain better light absorption. Figure 5(b) depicts the absorption enhancement for various particle sizes $(D)$ and periods $(P)$. The dash line indicates the position of optimal size and period. It visually demonstrates the relation between enhancement factor and SNP parameters (particle size $D$ and period $P$ ) and the relation is summarized as in the following empirical formula:

$$
P_{\text {optimum }}=\frac{17}{2} \cdot D_{\text {optimum }}-55
$$

in which $P_{\text {optimum }}$ and $D_{\text {optimum }}$ refer to the optimal values to obtain highest absorption enhancement.

3.3. OSCs Embedded with Periodic SNCs. In previous works, it is found that the localized electric field intensity near metallic nanoparticle surface can be enhanced significantly when nanoparticles get close to neighboring particles $[24,30]$. It is because the overlapped plasmon gap modes distribute mainly at the gap regions or sharp corner area of the adjacent nanoparticles. Therefore, in this section nanochain structures composed of adjacent SNPs are used in OSC to enhance the light absorption.

In the simulation, SNCs including dimmer, trimmer, tetramer, and pentamer, composed of two, three, four, and five SNPs, are used as the light absorption enhancement structures. Thickness of active layer and diameter of SNP (as the unit in SNCs) are $60 \mathrm{~nm}$ and $10 \mathrm{~nm}$. The periods for dimmer, trimmer, tetramer and pentamer are 40 nanometers, 60 nanometers, 80 nanometers, and 100 nanometers, respectively. These parameters keep the ratio of period to SNCs length as a constant in every case. For comparison, OSC embedded with periodic monodisperse SNPs was also calculated. The light absorptions with these nanostructures are shown in Figure 6. Comparing to OSC embedded with monodisperse SNPs and the pristine one, we can find that periodic SNCs can significantly boost the light absorption of OSCs when the wavelength is longer than $550 \mathrm{~nm}$, especially in the long wavelength range of $650-800 \mathrm{~nm}$ where OSCs with monodisperse SNPs have a weak absorption. This result is contributed to the coupling effect of LSPRs excited on individual SNPs that would cause more powerful electric field around adjacent region in SNCs comparing to monodisperse SNPs.

To quantify the light absorption enhancement of OSCs with SNCs, we also achieved the integrating absorption in the wavelength region of $300-800 \mathrm{~nm}$ under AM1.5G irradiation condition. The factors of relative enhancement with the five types of nanostructures (monodisperse SNP, dimmer, trimmer, tetramer, and pentamer) are calculated as 3.13\%, $29.1 \%, 35.5 \%, 39.4 \%$, and $36.1 \%$, respectively. The behaviors of significant absorption enhancement for SNCs can be ascribed to significant light trapping in the long wavelength range. 


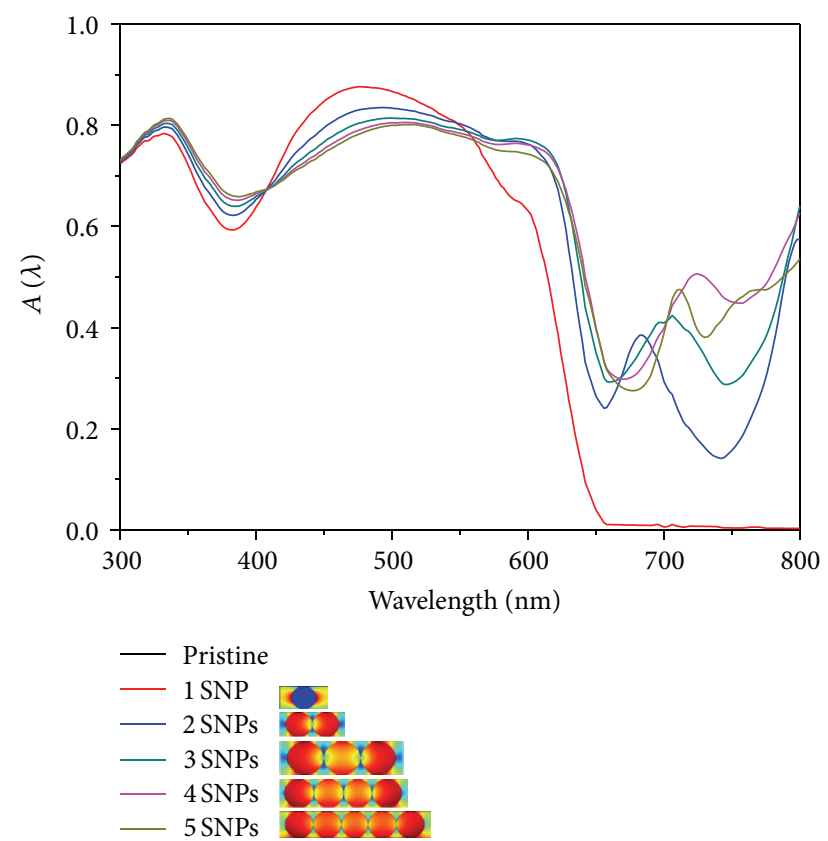

Figure 6: (Color online) Comparison of light absorption in various OSCs embedded with monodisperse SNPs and different type of SNCs.

\section{Conclusion}

In conclusion, we presented a detailed numerical investigation on the plasmonic OSCs embedded with periodic SNPs and SNCs using a finite element method. We analyzed the enhancement mechanism of light absorption and systematically studied the influences of factors such as the size and the period of SNPs on OSCs. The optimal parameters of $D=30 \mathrm{~nm}$ and $P=200 \mathrm{~nm}$ were found and the highest relative enhancement of $107.1 \%$ was obtained for periodic monodisperse SNPs. Meanwhile, we compared SNPs and SNCs to light absorption in OSCs, and we found that the SNCs can obtain more absorption enhancement because of the strong coupling effect of LSPRs.

\section{Conflict of Interests}

The authors declare that there is no conflict of interests regarding the publication of this paper.

\section{Acknowledgments}

This work is supported by NSFC under Grant nos. 61307066 and 21173041, Doctoral Fund of Ministry of Education of China under Grant nos. 20110092110016 and 20130092120024 , Graduate Innovation Program of Jiangsu Province under Grant no. CXLX_0114, Natural Science Foundation of Jiangsu Province under Grant no. BK20130630, the National Basic Research Program of China (973 Program) under Grant no. 2011CB302004, and the Foundation of Key Laboratory of Micro-Inertial Instrument and Advanced Navigation
Technology, Ministry of Education, China under Grant no. 201204.

\section{References}

[1] N. S. Sariciftci, L. Smilowitz, A. J. Heeger, and F. Wudl, "Photoinduced electron transfer from a conducting polymer to buckminsterfullerene," Science, vol. 258, no. 5087, pp. 1474-1476, 1992.

[2] G. Yu, J. Gao, J. C. Hummelen, F. Wudl, and A. J. Heeger, "Polymer photovoltaic cells: enhanced efficiencies via a network of internal donor-acceptor heterojunctions," Science, vol. 270, no. 5243, pp. 1789-1791, 1995.

[3] Y. K. Jin, K. Lee, N. E. Coates et al., "Efficient tandem polymer solar cells fabricated by all-solution processing," Science, vol. 317, no. 5835, pp. 222-225, 2007.

[4] H.-Y. Chen, J. Hou, S. Zhang et al., "Polymer solar cells with enhanced open-circuit voltage and efficiency," Nature Photonics, vol. 3, no. 11, pp. 649-653, 2009.

[5] Z. Wang, P. Han, H. Lu et al., "Advanced PERC and PERL production cells with $20.3 \%$ record efficiency for standard commercial p-type silicon wafers," Progress in Photovoltaics: Research and Applications, vol. 20, no. 3, pp. 260-268, 2012.

[6] C. Algora, E. Ortiz, I. Rey-Stolle et al., "A GaAs solar cell with an efficiency of $26.2 \%$ at 1000 suns and $25.0 \%$ at 2000 suns," IEEE Transactions on Electron Devices, vol. 48, no. 5, pp. 840-844, 2001.

[7] S. Ishizuka, H. Hommoto, N. Kido, K. Hashimoto, A. Yamada, and S. Niki, "Efficiency enhancement of $\mathrm{Cu}(\mathrm{In}, \mathrm{Ga}) \mathrm{Se} 2$ solar cells fabricated on flexible polyimide substrates using alkalisilicate glass thin layers," Applied Physics Express, vol. 1, no. 9, Article ID 092303, 3 pages, 2008.

[8] S. Sista, M.-H. Park, Z. Hong et al., "Highly efficient tandem polymer photovoltaic cells," Advanced Materials, vol. 22, no. 3, pp. 380-383, 2010.

[9] M.-H. Chen, J. Hou, Z. Hong et al., "Efficient polymer solar cells with thin active layers based on alternating polyfluorene copolymer/fullerene bulk heterojunctions," Advanced Materials, vol. 21, no. 42, pp. 4238-4242, 2009.

[10] S. H. Park, A. Roy, S. Beaupré et al., "Bulk heterojunction solar cells with internal quantum efficiency approaching $100 \%$," Nature Photonics, vol. 3, no. 5, pp. 297-303, 2009.

[11] D. E. Markov, C. Tanase, P. W. M. Blom, and J. Wildeman, "Simultaneous enhancement of charge transport and exciton diffusion in poly(p-phenylene vinylene) derivatives," Physical Review B, vol. 72, no. 4, Article ID 045217, 2005.

[12] P. E. Shaw, A. Ruseckas, and I. D. W. Samuel, "Exciton diffusion measurements in poly(3-hexylthiophene)," Advanced Materials, vol. 20, no. 18, pp. 3516-3520, 2008.

[13] J. Y. Kim, S. H. Kim, H.-H. Lee et al., "New architecture for high-efficiency polymer photovoltaic cells using solution-based titanium oxide as an optical spacer," Advanced Materials, vol. 18, no. 5, pp. 572-576, 2006.

[14] D. Kozanoglu, D. H. Apaydin, A. Cirpan, and E. N. Esenturk, "Power conversion efficiency enhancement of organic solar cells by addition of gold nanostars, nanorods, and nanospheres," Organic Electronics, vol. 14, no. 7, pp. 1720-1727, 2013.

[15] I. Diukman, L. Tzabari, N. Berkovitch, N. Tessler, and M. Orenstein, "Controlling absorption enhancement in organic photovoltaic cells by patterning Au nano disks within the active layer," Optics Express, vol. 19, no. 1, pp. A64-A71, 2011. 
[16] J.-Y. Lee and P. Peumans, "The origin of enhanced optical absorption in solar cells with metal nanoparticles embedded in the active layer," Optics Express, vol. 18, no. 10, pp. 10078-10087, 2010.

[17] A. J. Morfa, K. L. Rowlen, T. H. Reilly, M. J. Romero, and J. Van De Lagemaat, "Plasmon-enhanced solar energy conversion in organic bulk heterojunction photovoltaics," Applied Physics Letters, vol. 92, Article ID 013504, 2008.

[18] M. N. Lin, S. Q. Zhu, X. Y. Zhang, T. Zhang, and D. Su, "Islandshaped gold nanoparticle film with controlable optical properties for surface enhanced Ramanscattering," NRL Advances. In press.

[19] M.-G. Kang, T. Xu, H. J. Park, X. Luo, and L. J. Guo, "Efficiency enhancement of organic solar cells using transparent plasmonic Ag nanowire electrodes," Advanced Materials, vol. 22, no. 39, pp. 4378-4383, 2010.

[20] M.-G. Kang, M.-S. Kim, J. Kim, and L. J. Guo, "Organic solar cells using nanoimprinted transparent metal electrodes," Advanced Materials, vol. 20, pp. 4408-4413, 2008.

[21] D. Duche, P. Torchio, L. Escoubas et al., "Improving light absorption in organic solar cells by plasmonic contribution," Solar Energy Materials and Solar Cells, vol. 93, no. 8, pp. 13771382, 2009.

[22] H. Shen, P. Bienstman, and B. Maes, "Plasmonic absorption enhancement in organic solar cells with thin active layers," Journal of Applied Physics, vol. 106, no. 7, Article ID 073109, 2009.

[23] B. Zimmermann, M. Glatthaar, M. Niggemann, M. K. Riede, A. Hinsch, and A. Gombert, "ITO-free wrap through organic solar cells-A module concept for cost-efficient reel-to-reel production," Solar Energy Materials and Solar Cells, vol. 91, no. 5, pp. 374-378, 2007.

[24] S. Q. Zhu, T. Zhang, X. L. Guo, Q. L. Wang, X. F. Liu, and X. Y. Zhang, "Gold nanoparticle thin films fabricated by electrophoretic deposition method for highly sensitive SERS application," Nanoscale Research Letters, vol. 7, no. 1, p. 613, 2012.

[25] F. Moreno, B. García-Cámara, J. M. Saiz, and F. González, "Interaction of nanoparticles with substrates: effects on the dipolar behaviour of the particles," Optics Express, vol. 16, no. 17, pp. 12487-12504, 2008.

[26] C.-H. Kim, S.-H. Cha, S. C. Kim et al., "Silver nanowire embedded in P3HT:PCBM for high-efficiency hybrid photovoltaic device applications," ACS Nano, vol. 5, no. 4, pp. 3319-3325, 2011.

[27] D. D. S. Fung, L. Qiao, W. C. H. Choy et al., "Optical and electrical properties of efficiency enhanced polymer solar cells with Au nanoparticles in a PEDOT-PSS layer," Journal of Materials Chemistry, vol. 21, no. 41, pp. 16349-16356, 2011.

[28] M. Sundberg, O. Inganäs, S. Stafström, G. Gustafsson, and B. Sjögren, "Optical absorption of poly(3-alkylthiophenes) at low temperatures," Solid State Communications, vol. 71, no. 6, pp. 435-439, 1989.

[29] X. Lu, M. Rycenga, S. E. Skrabalak, B. Wiley, and Y. Xia, "Chemical synthesis of novel plasmonic nanoparticles," Annual Review of Physical Chemistry, vol. 60, pp. 167-192, 2009.

[30] T. Zhang, X. Y. Zhang, X. J. Xue, X. F. Wu, C. Li, and A. Hu, "Plasmonic properties of welded metal nanoparticles," Open Surface Science Journal, vol. 3, pp. 76-81, 2011. 

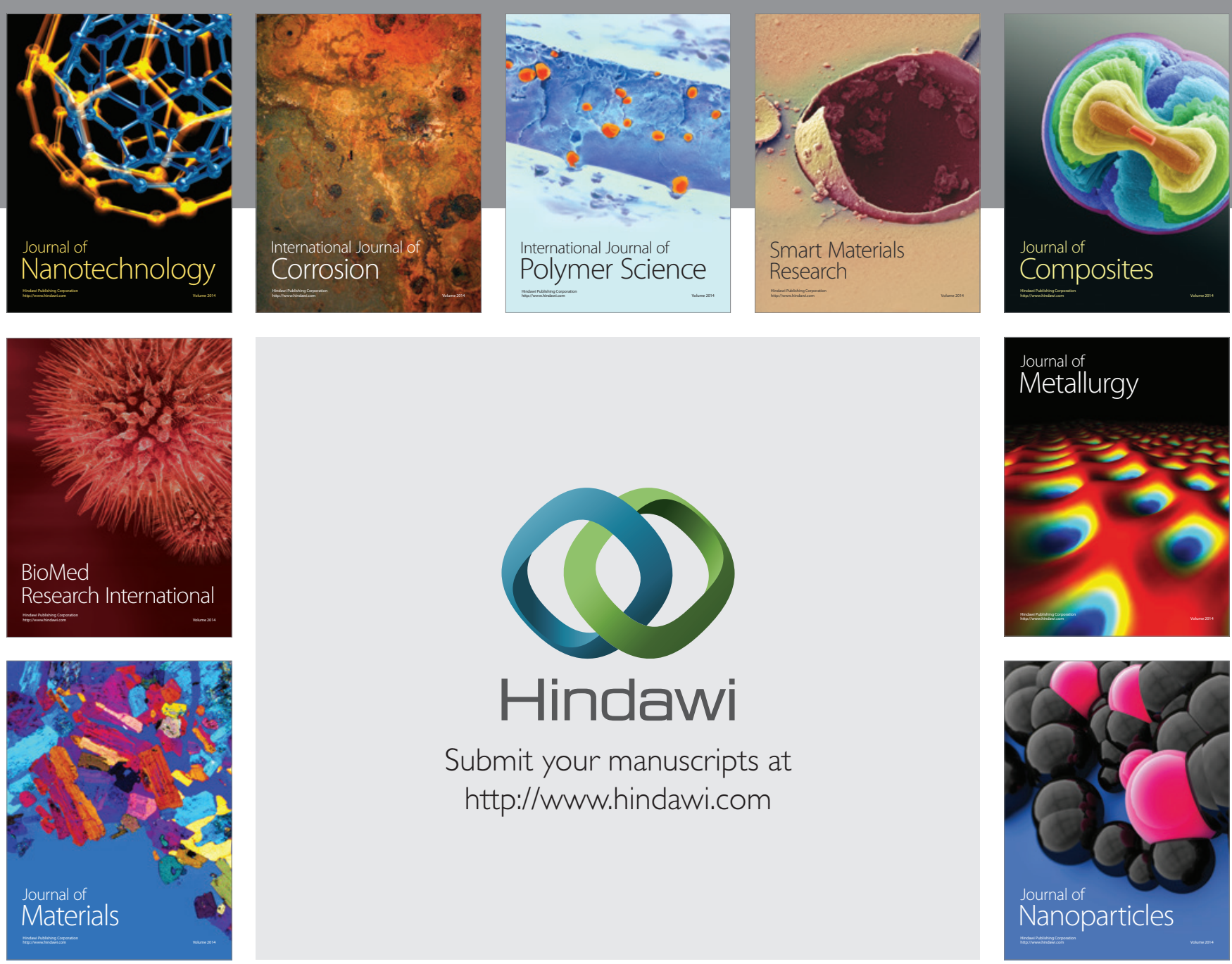

Submit your manuscripts at http://www.hindawi.com
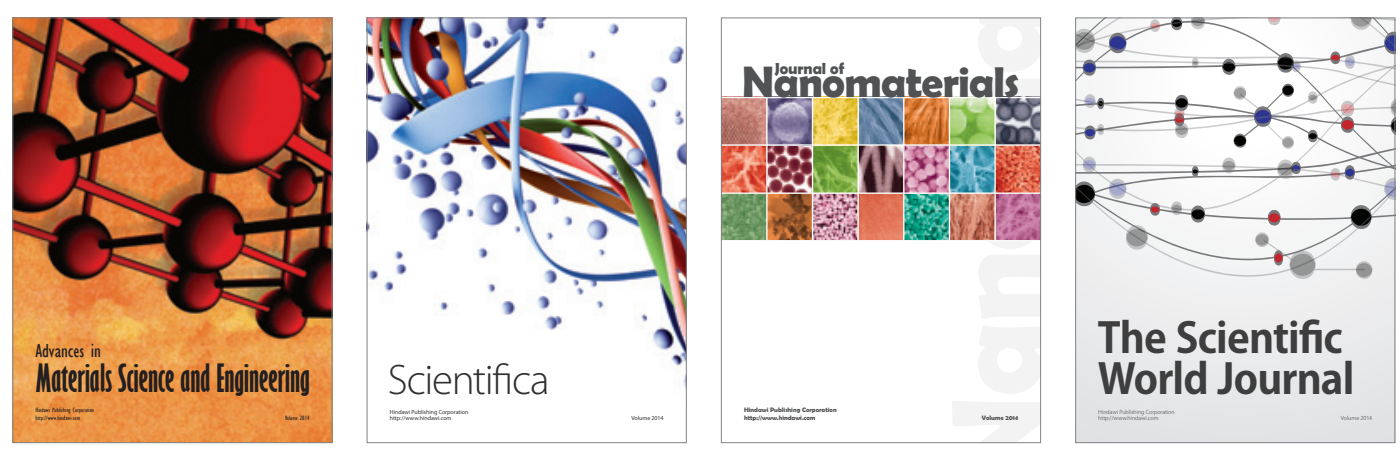

\section{The Scientific World Journal}
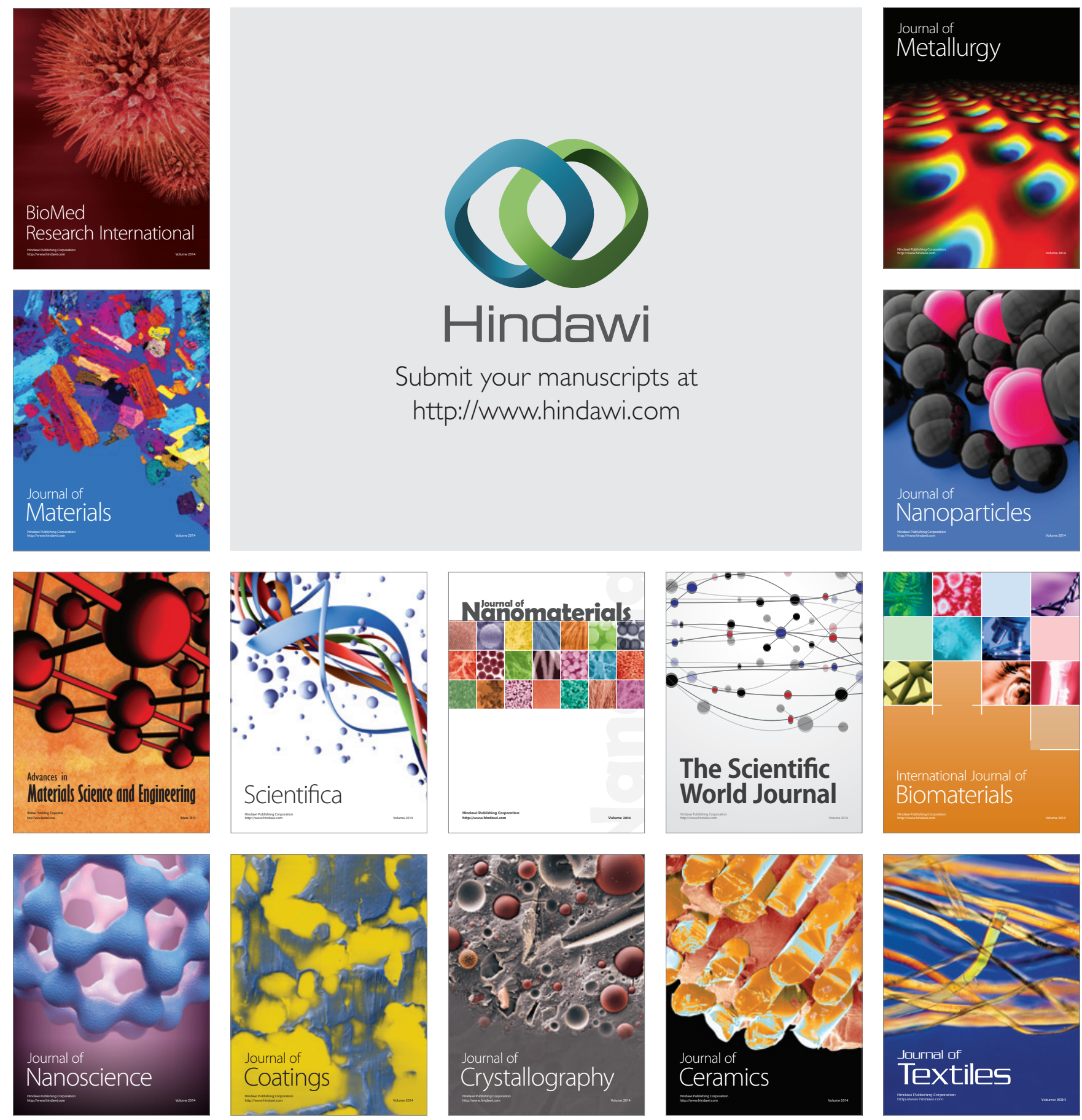